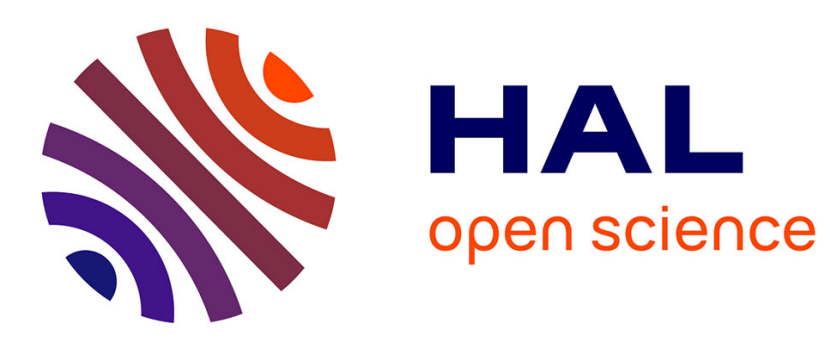

\title{
Modeling the mechanical response of polycrystals deforming by climb and glide
}

Ricardo A. Lebensohn, Craig S. Hartley, Carlson N. Tomé, Olivier Castelnau

\section{To cite this version:}

Ricardo A. Lebensohn, Craig S. Hartley, Carlson N. Tomé, Olivier Castelnau. Modeling the mechanical response of polycrystals deforming by climb and glide. Philosophical Magazine, 2010, 90 (5), pp.567583. 10.1080/14786430903213320. hal-02625454

\section{HAL Id: hal-02625454 https://hal.science/hal-02625454}

Submitted on 26 May 2020

HAL is a multi-disciplinary open access archive for the deposit and dissemination of scientific research documents, whether they are published or not. The documents may come from teaching and research institutions in France or abroad, or from public or private research centers.
L'archive ouverte pluridisciplinaire HAL, est destinée au dépôt et à la diffusion de documents scientifiques de niveau recherche, publiés ou non, émanant des établissements d'enseignement et de recherche français ou étrangers, des laboratoires publics ou privés. 


\title{
Modeling the mechanical response of polycrystals deforming by climb and glide
}

\author{
Ricardo A. Lebensohn ${ }^{a *}$, Craig S. Hartley ${ }^{\mathrm{b}}$, \\ Carlos N. Tomé ${ }^{\mathrm{a}}$ and Olivier Castelnau ${ }^{\mathrm{c}}$ \\ ${ }^{a}$ Materials Science and Technology Division, Los Alamos National Laboratory, \\ MS G755, Los Alamos, NM 87545, USA ${ }^{b}$ El Arroyo Enterprises LLC, \\ 231 Arroyo Sienna Drive, Sedona, AZ 86336, USA; ${ }^{c}$ Laboratoire des \\ Proprietés Mécaniques et Thermodynamiques des Matériaux, \\ Université Paris XIII, Av. J.-B.Clement, 93430 Villetaneuse, France
}

\begin{abstract}
This paper presents a crystallographically-based constitutive model of a single crystal deforming by climb and glide. The proposed constitutive law is an extension of the rate-sensitivity approach for single crystal plasticity by dislocation glide. Based on this description at single crystal level, a homogenization-based polycrystal model for aggregates deforming in a climb-controlled thermal creep regime is developed. To illustrate the capabilities of the proposed model, we present calculations of effective behavior of olivine and texture evolution of aluminum at warm temperature and low strain rate. In both cases, the addition of climb as a complementary single-crystal deformation mechanism improves the polycrystal model predictions.
\end{abstract}

Keywords: constitutive equations; creep; micromechanics; plasticity of crystals; texture

\section{Introduction}

At high homologous temperatures and stresses above a certain threshold, materials exhibit rate-dependent plastic deformation, carried by stress-assisted and thermally activated dislocation motion. Although, even at lower temperatures, the flow stress also shows some dependence with strain rate, this dependence becomes stronger above $0.3-0.4 T_{\mathrm{M}}$, determining a thermal power-law creep regime with low creep exponent (high rate sensitivity) [1]. This high-temperature plasticity regime can be further partitioned, depending on the actual microscopic mechanism that controls the dynamics of deformation. In metals below $0.5 T_{\mathrm{M}}$, a thermally and stress-assisted movement of dislocations over obstacles is responsible for a glide-controlled creep regime [1-3]. In this case, the shear component of the applied stress acting along the glide direction on the glide plane is the one that promotes dislocation movement. The creep exponent corresponding to this regime can be shown to be around 3 [1-3]. At higher temperatures, the interaction of dislocations with local non-equilibrium

\footnotetext{
*Corresponding author. Email: lebenso@1anl.gov
} 
concentrations of point defects permits the dislocations to climb as well as glide. Thus, there are two main differences between this climb-controlled creep $[1,4,5]$ and the previous glide-controlled regime. First, climb requires us to account for diffusion of point defects to or from a climbing dislocation. Secondly, in addition to the role played by the resolved shear stress, the effect of the diagonal stress components must also be accounted for. The creep exponent corresponding to the climb regime turns out to be close to 3 when lattice diffusion is the dominant mechanism $[1,4,5]$. In the special case of irradiated materials, the continuous creation of point defects during irradiation affects the dynamics of climb-controlled creep. When materials under irradiation are subjected to stresses well below their flow stress, they deform by irradiation creep at any temperature, with strain rates that are proportional to the applied stress (linear creep) [6-8]. For materials irradiated at high temperature, irradiation and thermal creep are coupled mechanisms. This coupling may lead to a significant increase on the total creep rate, compared to the mere additive superposition of both creep mechanisms [9].

Despite the relevant role played by dislocation climb in high-temperature plasticity and irradiation creep, the present work is, to our knowledge, one of the few attempts (and the most comprehensive) to consider the simultaneous activity of crystallographic climb and glide, and to utilize the resulting continuum constitutive description to model the behavior of polycrystalline aggregates deforming in the climb-controlled creep regime. Previous works that made use of the geometry of climb at single crystal level to construct a polycrystal model are the formulations of Rougier et al. [10,11] and Tomé and Christodoulou [12] for the study of the irradiation-induced creep and growth of $\mathrm{Zr}$ alloy polycrystals.

The paper is organized as follows. In Section 2, we present the constitutive description of a single crystal deforming viscoplastically by climb and glide, taking into account the slip-system geometry and the character of the dislocations present in the crystal. The proposed constitutive behavior is an extension of the ratesensitivity approach for single crystal plasticity by dislocation glide. In Section 3, we perform a limit analysis for the cases in which the dislocations in the crystal show either a pure edge or a pure screw character. In Section 4, we show how a polycrystal model based on non-linear homogenization can be built from the climb-and-glide constitutive description for single crystals. In Section 5, we present two examples of high-temperature plasticity for a geological material (olivine) and a metallic alloy (aluminum). We show that the addition of climb as complementary deformation mechanism at single crystal level improves the predictions of our homogenizationbased polycrystal model. In Section 6, we provide some concluding remarks and envisage future applications of the proposed model.

\section{Single crystal deformation by climb and glide}

For applied stresses above a certain threshold, plastic deformation occurs largely by dislocations overcoming obstacles under the influence of stress-assisted thermal activation. In what follows, we will assume stresses applied to a crystalline material that are above the threshold (otherwise, the deformation process would be controlled by pure diffusional flow, without a significant role of the dislocations). The geometry 


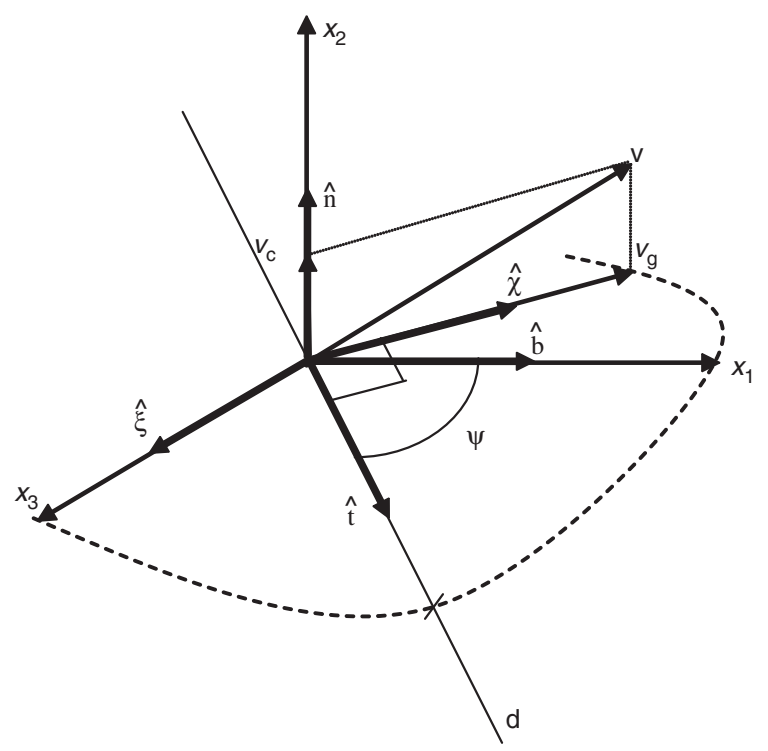

Figure 1. Slip coordinate system and corresponding unit vectors $(\hat{\mathbf{b}}, \hat{\mathbf{n}}, \hat{\boldsymbol{\xi}})$. Unit vectors of the dislocation coordinate system $(\hat{\mathbf{n}}, \hat{\mathbf{t}}, \hat{\boldsymbol{\chi}})$. Dislocation line and dislocation velocity are also shown.

of the above process can be described most simply in terms of the motion of a single dislocation of arbitrary orientation [13]. Let us consider the coordinate system $\left(x_{1}, x_{2}, x_{3}\right)$ (Figure 1). The normal to the slip plane is specified by the unit vector $\hat{\mathbf{n}}$ (in what follows, the caret is used to indicate unit vectors) along direction $x_{2}$. Dislocation motion produces a displacement $\mathbf{b}$ (Burgers vector) of the crystal containing $+\hat{\mathbf{n}}$ relative to that containing $-\hat{\mathbf{n}}$ in the direction of the unit vector $\hat{\mathbf{b}}$ along $x_{1}$. This slip coordinate system is completed by the unit vector $\hat{\xi}=\hat{\mathbf{b}} \times \hat{\mathbf{n}}$ along direction $x_{3}$, the Taylor axis.

Dislocation motion occurs in the direction of the velocity vector $\mathbf{v}$, which is normal to the dislocation line and has two components: the glide velocity, $v_{g}$, which lies in the slip plane, and the climb velocity, $v_{c}$, which is parallel (or anti-parallel) to $\hat{\mathbf{n}}$. Since a screw dislocation has no unique slip plane, the velocity vector of a screw dislocation can lie in any plane on which the dislocation can glide. The slip plane of a moving screw dislocation is specified by its unit normal, formed by the vector product of unit vectors parallel to $\mathbf{v}$ and $\mathbf{b}$. Since it must lie entirely in a slip plane, the velocity vector for a screw dislocation has no climb component.

Another reference frame, the dislocation coordinate system, can be defined based on $\hat{\mathbf{t}}$, the tangent to the dislocation line, $\hat{\mathbf{n}}$, and $\hat{\chi}$, a unit vector parallel to $v_{g}$. Both the glide and climb velocity of these components arise in response to the corresponding component of the Peach-Koehler (P-K) force on the dislocation. The Peach-Koehler force, a virtual force per unit length acting on the dislocation, is defined as $[14,15]$

$$
\mathbf{f}=(\sigma \cdot \mathbf{b}) \times \hat{\mathbf{t}}
$$


from which we can define a glide component $f_{g}=\mathbf{f} \cdot \hat{\chi}$, and a climb component $f_{c}=\mathbf{f} \cdot \hat{\mathbf{n}}$. Note that, by this definition, the force on a moving screw dislocation is purely a glide force, since it always lies in its glide plane. Hence, motion of the screw dislocation in response to the $\mathrm{P}-\mathrm{K}$ force is always conservative, i.e. it occurs without the emission or absorption of point defects.

Replacing Equation (1) into these definitions gives

$$
\begin{gathered}
f_{g}=[(\sigma \cdot \mathbf{b}) \times \hat{\mathbf{t}}] \cdot \hat{\chi}=(\sigma \cdot \mathbf{b}) \cdot(\hat{\mathbf{t}} \times \hat{\chi})=|\mathbf{b}| \sigma:(\hat{\mathbf{b}} \otimes \hat{\mathbf{n}})=|\mathbf{b}| \sigma^{\prime}:(\hat{\mathbf{b}} \otimes \hat{\mathbf{n}}) \\
f_{c}=[(\sigma \cdot \mathbf{b}) \times \hat{\mathbf{t}}] \cdot \hat{\mathbf{n}}=(\sigma \cdot \mathbf{b}) \cdot(\hat{\mathbf{t}} \times \hat{\mathbf{n}})=-|\mathbf{b}| \sigma:(\hat{\mathbf{b}} \otimes \hat{\chi}) .
\end{gathered}
$$

Note that the last equality in Equation (2) follows from the normality between $\hat{\mathbf{b}}$ and $\hat{\mathbf{n}}$, and states that the glide force depends only on the deviatoric component of the stress, $\sigma^{\prime}$. The climb force, on the other hand, depends on the full Cauchy stress, $\sigma$. However, further thermodynamic considerations (see [16,17] for details) led to a modification of Equation (1), involving the distinction between a mechanical force produced by an applied stress, originally identified by Peach and Koehler [14] and a chemical force due to non-equilibrium concentration of vacancies, originally proposed by Bardeen and Herring [18], such that [16]

$$
\mathbf{f}=\left(\sigma^{\prime} \cdot \mathbf{b}\right) \times \hat{\mathbf{t}}+\left[-\frac{k_{B} T}{\alpha|\mathbf{b}|^{3}} \log \left(x_{v} / x_{v}^{o, P T}\right)\right] \mathbf{b} \times \hat{\mathbf{t}},
$$

where $\alpha|\mathbf{b}|^{3}$ is the volume change due to the formation of a vacancy and the first and second terms on the right are the mechanical, or Peach-Koehler, and chemical, or Bardeen-Herring, components of the force, respectively. In the expression for the chemical force, $x_{v}$ is the actual concentration of vacancies, $x_{v}^{o, P T}$ is the equilibrium concentration of vacancies at a hydrostatic pressure $P$ and temperature $T$, and $k_{B}$ is Boltzmann's constant. Therefore, while the expression of the glide component of the force is still given by

$$
f_{g}=\left[\left(\sigma^{\prime} \cdot \mathbf{b}\right) \times \hat{\mathbf{t}}\right] \cdot \hat{\chi}=|\mathbf{b}| \sigma^{\prime}:(\hat{\mathbf{b}} \otimes \hat{\mathbf{n}}),
$$

the climb component should be written as

$$
\begin{aligned}
f_{c} & =\left[\left(\sigma^{\prime} \cdot \mathbf{b}\right) \times \hat{\mathbf{t}}+\left[-\frac{k_{B} T}{\alpha|\mathbf{b}|^{3}} \log \left(x_{v} / x_{v}^{o, P T}\right)\right] \mathbf{b} \times \hat{\mathbf{t}}\right] \cdot \hat{\mathbf{n}} \\
& =-|\mathbf{b}| \sigma^{\prime}:(\hat{\mathbf{b}} \otimes \hat{\chi})-|\mathbf{b}|\left[-\frac{k_{B} T}{\alpha|\mathbf{b}|^{3}} \log \left(x_{v} / x_{v}^{o, P T}\right)\right](\hat{\mathbf{b}} \otimes \hat{\chi}) .
\end{aligned}
$$

If the vacancy concentration in the crystal is everywhere equal to the equilibrium concentration for pressure $P$ and temperature $T$, the chemical force becomes zero and the pressure dependence of the climb force disappears. This condition is possible if the sources and sinks for vacancies (dislocations and grain boundaries) are such that vacancies are produced or annihilated by these sources and sinks to maintain the required equilibrium concentration. In the remainder of this paper, we will assume this condition is satisfied and will consider that the climb component is thus given by

$$
f_{c}=\left[\left(\sigma^{\prime} \cdot \mathbf{b}\right) \times \hat{\mathbf{t}}\right] \cdot \hat{\mathbf{n}}=-|\mathbf{b}| \sigma^{\prime}:(\hat{\mathbf{b}} \otimes \hat{\chi})
$$


Note that, in the case of the glide component, Equation (5) is equivalent to Equation (2), while for the climb component, the Cauchy stress appearing in Equation (3) is replaced in Equation (7) by the deviatoric stress.

The dyadic product in Equation (5) is the classic glide tensor, which can be decomposed into symmetric and antisymmetric parts. Using explicit index notation

$$
g_{i j}=\hat{b}_{i} \hat{n}_{j}=m_{i j}+q_{i j}=\frac{1}{2}\left(\hat{b}_{i} \hat{n}_{j}+\hat{b}_{j} \hat{n}_{i}\right)+\frac{1}{2}\left(\hat{b}_{i} \hat{n}_{j}-\hat{b}_{j} \hat{n}_{i}\right),
$$

where $m_{i j}$ is the symmetric Schmid tensor and $q_{i j}$ is the corresponding antisymmetric (plastic rotation) tensor (note that since $\hat{\mathbf{b}} \perp \hat{\mathbf{n}}, g_{i j}$ and $m_{i j}$ are traceless). The product $\sigma^{\prime}:(\hat{\mathbf{b}} \otimes \hat{\mathbf{n}})=m: \sigma^{\prime}$ appearing in Equation (5) is the resolved shear stress (i.e. the $\sigma_{12}^{\prime}$ component of the external stress tensor expressed in slip-system coordinates), which, according to the Schmid law, has to reach a threshold value for slip activation. Equation (5) thus provides a physical interpretation of the phenomenological Schmid law, i.e. slip is activated when a critical value of the force acting on dislocations is reached to overcome the slip resistance and start dislocation motion. The Schmid law has been modified to account for rate effects and multislip, giving raise to the widely used rate-sensitivity constitutive equation for single crystal material points deforming plastically by glide [19], i.e.

$$
\dot{\gamma}^{s}=\dot{\gamma}_{o}\left(\frac{\tau^{s}}{\tau_{o}^{s}}\right)^{n_{g}}=\dot{\gamma}_{o}\left(\frac{\left|m^{s}: \sigma^{\prime}\right|}{\tau_{o}^{s}}\right)^{n_{g}} \operatorname{sgn}\left(m^{s}: \sigma^{\prime}\right)
$$

and

$$
\dot{\varepsilon}_{i j}^{g}=\sum_{s=1}^{N_{s}} m_{i j}^{s} \dot{\gamma}^{s}=\dot{\gamma}_{o} \sum_{s=1}^{N_{s}} m_{i j}^{s}\left(\frac{\left|m^{s}: \sigma^{\prime}\right|}{\tau_{o}^{s}}\right)^{n_{g}} \operatorname{sgn}\left(m^{s}: \sigma^{\prime}\right),
$$

where $\dot{\varepsilon}^{g}$ is the (traceless) plastic strain rate tensor induced by glide. In the expression above, the sum runs over all $N_{\mathrm{s}}$ active slip systems; $m^{s}$ and $\tau_{o}^{s}$ are, respectively, the Schmid tensor and the threshold resolved shear stress associated with glide in system (s); $\dot{\gamma}^{s}$ is the shear rate on slip system (s); $\dot{\gamma}_{o}$ is a normalization factor and $n_{g}$ is the stress exponent (inverse of the rate-sensitivity) associated with glide. Note that, at sufficiently high stresses, the power-law Equation (9) is in fact an approximation of the actual rate-equation for dislocation glide limited by discrete obstacles, i.e. an Arrhenius relationship between the flow shear stress and the shear rate of the form $[1,20]$ :

$$
\dot{\gamma}^{s}=\dot{\gamma}^{o} \exp \left\{-\frac{\Delta F}{k T}\left(1-\frac{\tau^{s}}{\tau_{o}^{s}}\right)\right\},
$$

where $\Delta F$ is the total free energy required to overcome an obstacle without aid of external stress. The constitutive parameters $n_{g}$ and $\tau_{o}^{s}$ are temperature-, strain-rateand microstructure-dependent. The plastic rotation rate of the crystallographic lattice is given by

$$
\dot{\omega}_{i j}^{p}=\sum_{s=1}^{N_{s}} q_{i j}^{s} \dot{\gamma}^{s}
$$


By analogy with the aforementioned constitutive description of a single crystal deforming plastically by glide, the dyadic product appearing in Equations (6) and (7) can be defined as a climb tensor [13], i.e.

$$
c_{i j}=\hat{b}_{i} \hat{\chi}_{j}=k_{i j}+r_{i j}=\frac{1}{2}\left(\hat{b}_{i} \hat{\chi}_{j}+\hat{b}_{j} \hat{\chi}_{i}\right)+\frac{1}{2}\left(\hat{b}_{i} \hat{\chi}_{j}-\hat{b}_{j} \hat{\chi}_{i}\right),
$$

where $k_{i j}$ and $r_{i j}$ are, respectively, the symmetric and antisymmetric parts of the climb tensor. According to Equation (7), in the case of equilibrium concentration of vacancies, the climb tensor resolves the stress into a climb component in a similar way that the glide tensor does for the glide component. However, there are some important differences between the two tensors. While $g_{i j}$ is formed from two unit vectors based on the crystallography of the slip process independently of the dislocation line orientation, $c_{i j}$ depends on the orientation (character) of the dislocation. This dependence can be expressed in terms of a single parameter $\psi$, i.e. the angle between $\hat{\mathbf{t}}$ and $\hat{\mathbf{b}}$. Noting that $\hat{\mathbf{t}}=\hat{\mathbf{b}} \cos \psi+\hat{\xi} \sin \psi$ and $\hat{\chi}=\hat{\mathbf{n}} \times \hat{\mathbf{t}}$, one can write [13]

$$
c_{i j}(\psi)=\left(\hat{b}_{i} \hat{b}_{j}\right) \sin \psi-\left(\hat{b}_{i} \hat{\xi}_{j}\right) \cos \psi,
$$

where the dyadic products are only functions of the slip-system crystallography, and the dependence of the climb tensor with the dislocation orientation is specified by $\psi$. This parameter accounts for the relative edge-screw character of the average dislocation density, and can be regarded as a measure of the ratio between the lengths of mobile dislocation lines projected parallel $\left(\rho_{\text {screw }}\right)$ and perpendicular $\left(\rho_{\text {edge }}\right)$ to the slip direction in the slip plane, such that $\operatorname{tg} \psi=\rho_{\text {edge }} / \rho_{\text {screw }}$. In addition, it is evident from Equation (14) that the climb tensor is never traceless for dislocation distributions having a non-zero edge component, i.e. $\sin \psi \neq 0$, while the glide tensor is always traceless by definition. Hence, the symmetric climb tensor can be further decomposed into a deviatoric and a hydrostatic component, i.e.

$$
k_{i j}=k_{i j}^{d}+k_{i j}^{h} .
$$

For further analysis, the deviatoric, hydrostatic and antisymmetric components of the climb tensor expressed in slip-system coordinates are [13]

$$
\begin{gathered}
k_{i j}^{d}=\frac{1}{6}\left[\begin{array}{ccc}
4 \sin \psi & 0 & 3 \cos \psi \\
0 & -2 \sin & 0 \\
3 \cos \psi & 0 & -2 \sin
\end{array}\right] \\
k_{i j}^{h}=\frac{1}{3}\left[\begin{array}{ccc}
\sin \psi & 0 & 0 \\
0 & \sin \psi & 0 \\
0 & 0 & \sin \psi
\end{array}\right] \\
r_{i j}=\frac{1}{2}\left[\begin{array}{ccc}
0 & 0 & \cos \psi \\
0 & 0 & 0 \\
-\cos & 0 & 0
\end{array}\right] .
\end{gathered}
$$

Carrying on the analogy with the constitutive description of glide, we propose to treat climb as being activated when the climb force acting on dislocations reaches 
a critical value. In the case of equilibrium concentration of vacancies (i.e. climb force given by Equation (7)), we can then define the scalar climb rate on system (s) as (c.f. Equation (9)):

$$
\dot{\beta}^{s}=\dot{\gamma}_{o}\left(\frac{\left|k^{d, s}: \sigma^{\prime}\right|}{\sigma_{o}^{s}}\right)^{n_{c}} \operatorname{sgn}\left(k^{d, s}: \sigma^{\prime}\right),
$$

where $n_{c}$ and $\sigma_{o}^{s}$ are, respectively, the creep exponent and a scalar threshold stress associated with climb. Note that the form of Equation (19) accurately represents the rate-equation associated with climb-controlled power-law creep. However, for higher stresses, at which the rate-equation becomes exponential (power-law breakdown regime [1,21]), Equation (19) should be regarded as a phenomenological approximation to the actual rate-equation, in the same way that Equation (9) for glide is, under certain conditions, an approximation of rate-equation Equation (11). This definition leads to the following total strain rate of a single crystal deforming solely by climb (c.f. Equation (10)):

$$
\dot{\varepsilon}_{i j}^{c}=\sum_{s=1}^{N_{s}} k_{i j}^{d, s} \dot{\beta}^{s}=\dot{\gamma}_{o} \sum_{s=1}^{N_{s}} k_{i j}^{d, s}\left(\frac{\left|k^{d, s}: \sigma^{\prime}\right|}{\sigma_{o}^{s}}\right)^{n_{c}} \operatorname{sgn}\left(k^{d, s}: \sigma^{\prime}\right)
$$

and the following plastic spin associated with climb (c.f. Equation (12)):

$$
\dot{\omega}_{i j}^{p}=\dot{\gamma}_{o} \sum_{s} r_{i j}^{s} \dot{\beta}^{s}
$$

Like their glide counterparts $\left(n_{g}\right.$ and $\left.\tau_{o}^{s}\right)$, parameters $n_{c}$ and $\sigma_{o}^{s}$ depend on temperature, strain rate and microstructure. The latter dependency, however, is more complex than in the case of glide, since not only the dynamics and interaction between dislocations determine the current value of those internal variables, but also the interaction between point defects and dislocations are also relevant in the determination of the climb constitutive parameters. Moreover, note that, because the relative importance of the point defect-dislocation interaction depends on the character of the mobile dislocations, the climb parameters are likely to be also dependent on the average character of the dislocation density. However, for the sake of simplicity, and lacking physical insight into this dependence, in this work we have assumed that $n_{c}$ and $\sigma_{o}^{s}$ do not depend on $\psi$.

\section{Limit cases}

Given the dependence of the constitutive relation for a single crystal deforming by climb with the average character of the dislocation distribution (through the parameter $\psi$ ), it is interesting to analyze the limit cases of pure edge and pure screw dislocations. In the case of an edge dislocation $(\psi=\pi / 2)$, climb produces strain (shape change) without lattice rotation (Equations (18) and (21)). Moreover, in slip-system coordinates, the contracted product $k^{d, s}: \sigma^{\prime}$ appearing in Equation (19) turns out to be $\sigma_{11}^{\prime}$, and

$$
\dot{\beta}^{s}=\dot{\gamma}_{o}\left(\frac{\left|\sigma_{11}^{\prime}\right|}{\sigma_{o}^{s}}\right)^{n_{c}} \operatorname{sgn}\left(\sigma_{11}^{\prime}\right) \quad \text { (edge dislocation). }
$$


Hence, in the case of a pure edge dislocation, $\sigma_{o}^{s}$ can be interpreted as a threshold stretching stress, corresponding to the deviatoric stress component acting along the direction of the Burgers vector, which provides the driving force for point defects to migrate towards the core of the dislocation and make it climb.

In the case of a screw dislocation, $\psi=0$ and, in slip-system coordinates, it is

$$
\begin{gathered}
k^{d, s}: \sigma^{\prime}=\sigma_{13} \\
\dot{\beta}^{s}=\dot{\gamma}_{o}\left(\frac{\left|\sigma_{13}\right|}{\sigma_{o}^{s}}\right)^{n_{c}} \operatorname{sgn}\left(\sigma_{13}\right) \quad \text { (screw dislocation), }
\end{gathered}
$$

and, using Equation (24) in Equation (20), the only non-zero component of the climb rate tensor turns out to be the shear $\dot{\varepsilon}_{13}^{c}$. Hence, in the case of a pure screw dislocation, $\sigma_{o}^{s}$ should be interpreted as the threshold shear stress for cross-slip on the plane that is normal to the original slip plane defined by $\hat{n}$. This motion of a screw dislocation out of the slip plane on which the slip system is based is simply glide on a different plane, not climb, which does not need to be assisted by point-defect motion. If the crystallography of slip does not permit slip of screw dislocations on planes normal to the original slip plane, the glide force on the screw dislocation must be resolved onto permissible slip planes containing the original slip direction to obtain a suitable threshold stress. In the following development, we consider the case described as a limiting case and do not investigate the detailed crystallography of glide of screw dislocations on different secondary planes for the examples studied.

\section{Polycrystal model}

The above climb-and-glide constitutive description at the level of a single-crystal material point can be used within the context of polycrystal models based on crystal plasticity to expand their applicability to the thermal creep regime, at high temperatures and low strain rates. Examples of these polycrystal models include full-field formulations such as the different flavors of the crystal-plasticity finite element method (CPFEM) [22-29] or the Fast Fourier Transform (FFT)-based approach [30-32], and mean-field formulations such as Taylor-type methods [33] or self-consistent (SC) homogenization methods. In what follows, we present the extension of the viscoplastic self-consistent (VPSC) formulation [34,35] for the prediction of the mechanical behavior of polycrystals with constituent grains deforming by climb-and-glide. The original version of the SC formulation for aggregates of grains deforming by glide was implemented numerically in the VPSC code [36]. In recent years, the VPSC code has experienced a variety of improvements and extensions and it is nowadays extensively used to simulate plastic deformation of polycrystalline aggregates and to interpret experimental evidence on metallic, geological and polymeric materials (for a review of current capabilities and material systems studied with VPSC, see [37]).

The self-consistent (SC) formulation was originally developed for linear elastic materials [38]. For aggregates deforming in the viscoplastic regime, several selfconsistent approximations were subsequently proposed. All these non-linear variants are based on the linear SC solution, previous use of ad hoc linearization schemes 
to approximate the non-linear mechanical behavior at single-crystal level. Here, we present the climb-and-glide extension of the first-order affine [39] VPSC formulation.

Adding the contributions of glide (Equation (10)) and climb (Equation (19)), the constitutive equation of a single crystal $(r)$ deforming by climb and glide is given by

$$
\begin{aligned}
\dot{\varepsilon}_{i j}^{(r)}=\dot{\gamma}_{o}[ & \sum_{s=1}^{N_{s}} m_{i j}^{s(r)}\left(\frac{\left|m^{s(r)}: \sigma^{(r)}\right|}{\tau_{o}^{s(r)}}\right)^{n_{g}} \operatorname{sgn}\left(m^{s(r)}: \sigma^{\prime(r)}\right) \\
& \left.+\sum_{s=1}^{N_{s}} k_{i j}^{d, s(r)}\left(\frac{\left|k^{d, s(r)}: \sigma^{\prime(r)}\right|}{\sigma_{o}^{s(r)}}\right)^{n_{c}} \operatorname{sgn}\left(k^{d, s(r)}: \sigma^{\prime(r)}\right)\right],
\end{aligned}
$$

where the climb and glide tensors and threshold stresses are assumed to be constant in the domain of single crystal $(r)$, and the strain rate and stress are average tensors in the $(r)$ domain. The linearized (affine) moduli of single crystal $(r)$ follow from writing Equation (25) in a pseudo-linear form:

$$
\dot{\varepsilon}^{(r)}=M_{a f f}^{(r)}: \sigma^{(r)}+\dot{\varepsilon}_{a f f}^{o(r)},
$$

where

$$
\begin{gathered}
M_{a f f}^{(r)}=\dot{\gamma}_{o}\left[n_{g} \sum_{s=1}^{N_{s}} \frac{m^{s(r)} \otimes m^{s(r)}}{\tau_{o}^{s(r)}}\left(\frac{\left|m^{s(r)}: \sigma^{\prime(r)}\right|}{\tau_{o}^{s(r)}}\right)^{n_{g}-1}\right. \\
\left.+n_{c} \sum_{s=1}^{N_{s}} \frac{k^{d, s(r)} \otimes k^{d, s(r)}}{\sigma_{o}^{s(r)}}\left(\frac{\left|k^{d, s(r)}: \sigma^{\prime(r)}\right|}{\sigma_{o}^{s(r)}}\right)^{n_{c}-1}\right] \\
\dot{\varepsilon}_{a f f}^{o(r)}=\dot{\gamma}_{o}\left[\left(1-n_{g}\right) \sum_{s=1}^{N_{s}} m_{i j}^{s(r)}\left(\frac{\left|m^{s(r)}: \sigma^{\prime(r)}\right|}{\tau_{o}^{s(r)}}\right)^{n_{g}} \operatorname{sgn}\left(m^{s(r)}: \sigma^{\prime(r)}\right)\right. \\
\left.+\left(1-n_{c}\right) \sum_{s=1}^{N_{s}} k_{i j}^{d, s(r)}\left(\frac{\left|k^{d, s(r)}: \sigma^{\prime(r)}\right|}{\sigma_{o}^{s(r)}}\right)^{n_{c}} \operatorname{sgn}\left(k^{d, s(r)}: \sigma^{\prime(r)}\right)\right] .
\end{gathered}
$$

From these equations, a SC formulation can be built in the standard way (for details, see [37]). We have implemented this extended viscoplastic self-consistent formulation in the VPSC code, incorporating climb as an additional mechanism at single crystal level. Note that the affine linearization is very suitable for the above extension, since it does not require a single stress exponent, as other formulations do (e.g. tangent or secant linearizations [37]). Thus, the affine model can be used for different climb and glide exponents.

\section{Examples}

\subsection{Creep of olivine}

Olivine is the mineral that comprises the majority of the Earth's upper mantle. The mantle is known to exhibit seismic (elastic) anisotropy, commonly attributed to lattice preferred orientation (LPO, i.e. crystallographic texture) of its constituent minerals. The development of LPO is due to plastic deformation of these minerals 
associated with large-scale convective flow. Olivine crystals exhibit orthorhombic symmetry and have a few systems available for glide-controlled creep (only three of them are linearly independent, short of the five independent glide systems needed to accommodate an arbitrary deformation imposed to the crystal). This reduced number of linearly independent glide systems leads to a very high viscoplastic anisotropy at the grain scale in olivine. Hence, until now, either a very crude homogenization scheme such as the equilibrium-based Sachs model [40,41], or the presence of an artificial hard glide mode [42-44] that provides the additional degrees of freedom, had to be assumed to perform crystal plasticity-based calculations on polycrystalline aggregates of this material. Although by assuming a sufficiently hard artificial glide mode, its relative activity in VPSC simulations can be reduced to small values, the predicted effective response is strongly dependent on the threshold stress of such hard mode (e.g. in the affine VPSC case, the effective stress of a random olivine polycrystal scales with $M^{0.75}$, where the contrast parameter $M$ is the ratio between the critical stresses of the artificial hard mode and the soft systems. In full-field calculations, the exponent of $M$ turns out to be 0.5 [37]). This undesirable dependence of the effective stress with the strength of the artificial hard mode prevents geophysicists from a reliable use of such a model in multiscale simulations of mantle's convection [45].

The incorporation of dislocation climb as an additional deformation mechanism (dislocation climb is likely to be active in mantle's olivine, deforming in natural conditions at low strain rates and high homologous temperatures, in the power-law climb-controlled creep regime [46]) addresses the above limitation. Figure 2 shows the effective stress predicted by the VPSC formulation (with and without climb at single crystal level) for an olivine polycrystal with random texture, as a function

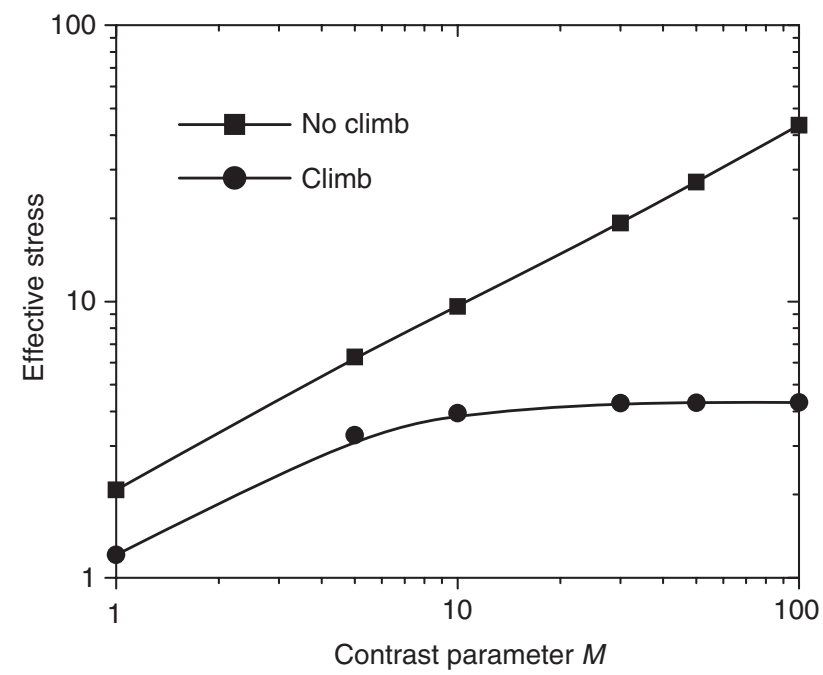

Figure 2. Effective stress of isotropic olivine versus contrast parameter at single crystal level predicted with the VPSC model assuming glide only and climb-and-glide as single crystal plastic deformation mechanisms. 
of the contrast parameter $M$, assuming the slip modes and constitutive parameters reported in Table 1. The slip modes and their correspondent threshold stresses for glide considered here are those used in [43], and based on experimental results [47-49]. As for the threshold stresses for climb, we have assumed them to be equal to their glide counterparts, and the edge and screw dislocation densities were assumed to be equal. Evidently, the incorporation of climb provides additional degrees of freedom to the available single crystal deformation mechanisms, such that the effective stress saturates at sufficiently high values of the contrast parameter. Figure 3 shows the relative activities (defined as the average strain-rates, calculated over the entire polycrystal, accommodated by each mechanism and each slip mode, divided by the total strain-rate) that were obtained in our climb-and-glide calculations versus the glide-only predictions (the relative activities of glide of all the soft systems were added together and shown under the 'glide' label, the relative

Table 1. Slip modes and corresponding climb-and-glide constitutive parameters for olivine single crystals.

\begin{tabular}{cccccc}
\hline Slip mode & $\tau_{o, g}$ & $n_{g}$ & $\tau_{o, c}$ & $n_{c}$ & $\Psi_{o}$ \\
\hline$(010)[100]$ & 1. & 3 & 1. & 3 & $\pi / 4$ \\
$(001)[100]$ & 1. & 3 & 1. & 3 & $\pi / 4$ \\
$(010)[001]$ & 2. & 3 & 2. & 3 & $\pi / 4$ \\
$(100)[001]$ & 3. & 3 & 3. & 3 & $\pi / 4$ \\
$\{111\}(110)$ & $\mathrm{M}$ & 3 & $\mathrm{M}$ & 3 & $\pi / 4$ \\
\hline
\end{tabular}
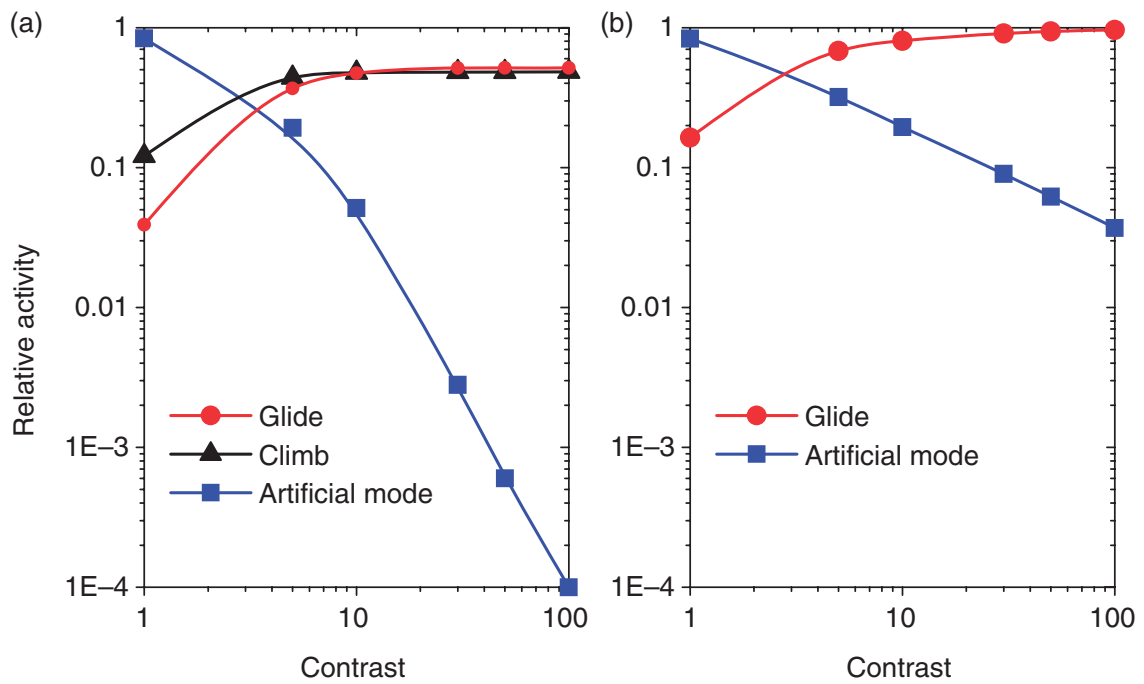

Figure 3. (a) Case of climb-and-glide in olivine. Relative activities of glide in the four slip modes observed in olivine, climb in the four observed slip modes, and climb-and-glide in the artificial mode. (b) Case of glide only. Relative activities of glide in the four observed slip modes and the fictitious mode. 
activities of climb of the same set of systems were added together under 'climb', and the glide and climb activities of the hard mode were added together under the 'artificial mode' label). Evidently, the availability of the climb mechanism drastically reduces the need for activation of the artificial mode when the contrast is large.

\subsection{Low strain-rate compression textures of Al at warm temperature}

Stout et al. [50] reported compression textures of a $5182 \mathrm{Al}$ alloy deformed up to $60 \%$ strain, at different temperatures (from room temperature to $550^{\circ} \mathrm{C}$ ) and strain rates $\left(1\right.$ and $\left.10^{-3} \mathrm{~s}^{-1}\right)$. Cylindrical samples were cut with their axes along the rolling and normal directions of a plate with a typical $(001)\langle 100\rangle$ recrystallization texture. Therefore, the compression samples had an initial texture with the (001) crystal orientations predominantly oriented along the compression axis, up to an intensity of about $4 \mathrm{mrd}$ (multiple of random distribution). The Stout et al. [50] results showed that, depending on the temperature and the strain rate of the test, the final textures were characterized by either a retained $(001)$ cube texture, or a $\langle 110\rangle$ fiber (i.e. a typical compression texture in fcc materials), or a mixed texture with both the (001) and (110) components present. Another important result reported in [50] is that the samples tested at $400^{\circ} \mathrm{C}$ and below showed no evidence of dynamic recrystallization during the compression test or static recrystallization during quenching. Samples tested at room temperature and $200^{\circ} \mathrm{C}$ at both strain rates showed typical (110) compression textures. At $400^{\circ} \mathrm{C}$ and a strain rate of $10^{-3} \mathrm{~s}^{-1}$, both the (001) and the (110) components are present, with similar intensity in the measured inverse pole figures, while at $400^{\circ} \mathrm{C}$ and $1 \mathrm{~s}^{-1}$, the $(001)$ cube component is greatly diminished. To explain the presence of the (001) cube component in absence of recrystallization, Stout et al. [50] invoked the activity of non-octahedral (110) $\langle 1 \overline{1} 0\rangle$ slip (together with the standard (111) $\langle 1 \overline{1} 0\rangle$ octahedral slip mode), reported to occur in experiments done on Al single crystal and polycrystals at such temperature [51], and whose activity is known to stabilize the cube component [52]. On the other hand, to explain the differences between the $400^{\circ} \mathrm{C}$ textures at different strain rates, Stout et al. [50] had to make a very strong assumption, namely that there is a very sharp decrease in rate sensitivity as strain rate increases.

However, the retained (001) cube component at warm temperature and low strain rate can be satisfactorily explained in terms of the likely activity of climb under those conditions, as follows. Figure 4 shows different VPSC simulations of texture evolution of Al 5182 after $60 \%$ uniaxial compression. Lines correspond to increments of $0.5 \mathrm{mrd}$ and dots indicate regions below $0.5 \mathrm{mrd}$. The initial (001) texture, with a maximum intensity of $4+\operatorname{mrd}$ is shown in Figure $4 \mathrm{a}$. The second row shows the influence of the rate-sensitivity exponent when glide only on slip mode (111) $\langle 110\rangle$ is assumed. Evidently, as pointed out in [50], an increase in rate sensitivity contributes to the retention of the cube component. However, even when a reasonably low creep exponent is used (i.e. $n=3$ ), the standard (110) compression texture component is more than twice as intense as the retained (001) cube component. (Note that, according to Frost and Ashby's deformation-mechanism map for $\mathrm{Al}$ [1], the combination of homologous temperature, strain rate and applied stress clearly implies that both $400^{\circ} \mathrm{C}$ tests lie within the climb-controlled creep 

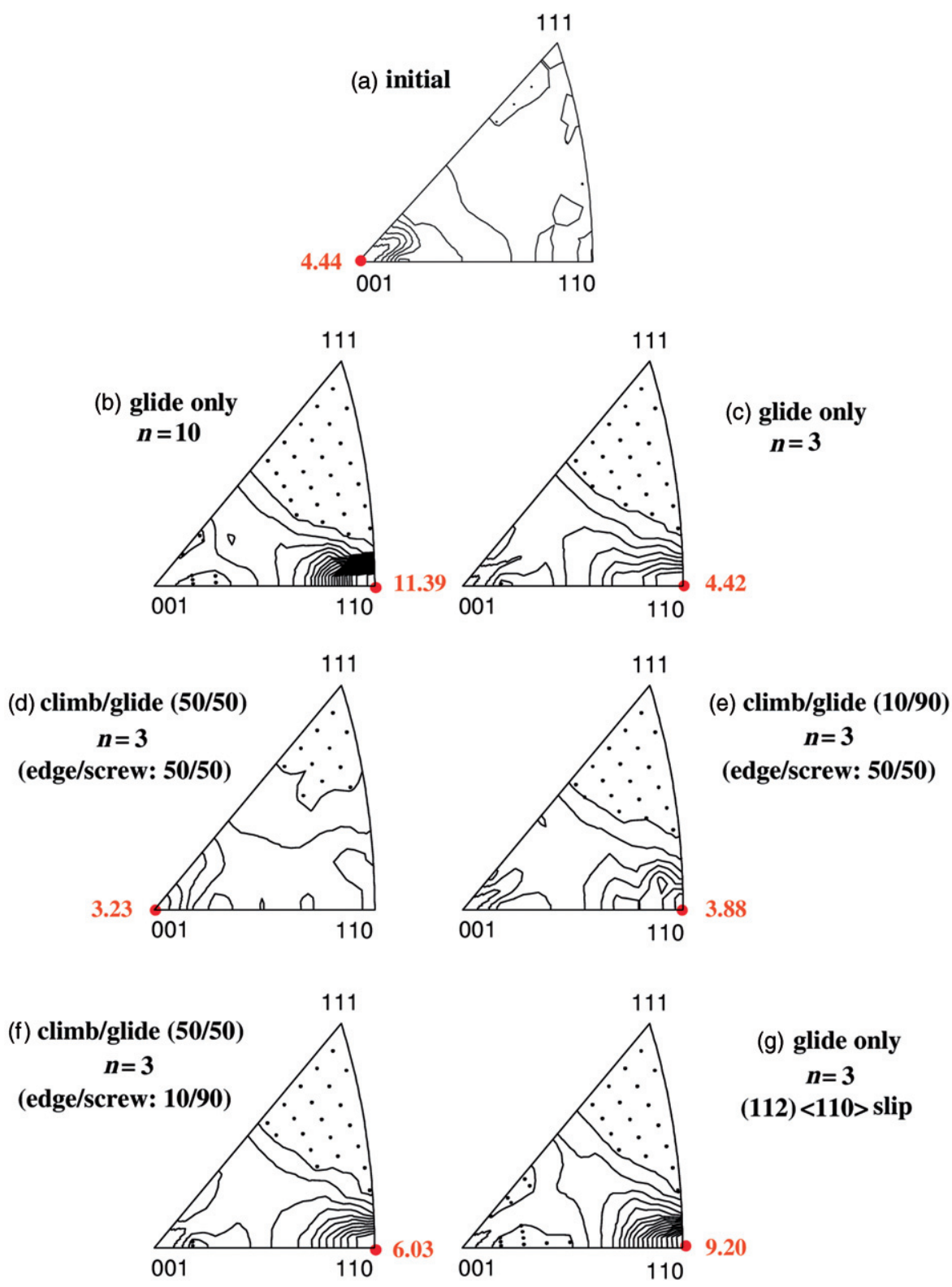

Figure 4. VPSC simulations of texture evolution of Al 5182 after $60 \%$ uniaxial compression. Lines correspond to increments of $0.5 \mathrm{mrd}$. Dots are regions below $0.5 \mathrm{mrd}$. First row: (a) initial (001) texture. Second row: (111) $\langle 110\rangle$ glide: (b) $n=10$; (c) $n=3$. Third row: climb and glide in (111) $\langle 110\rangle, n=3$, equal edge and screw dislocation densities $(\psi=\pi / 4)$ : (d) $\sigma_{o}^{s}=\tau_{o}^{s}$; (e) $\sigma_{o}^{s}=2 \times \tau_{o}^{s}$. Fourth row: (f) climb and glide in (111) $\langle 110\rangle, n=3$, predominant screw dislocation density $(\psi=0.01)$; (g) (112) $\langle 110\rangle$ glide, $n=3$. 
regime, characterized by an exponent not larger than 4.) The third row shows the VPSC calculations considering climb and glide activity at grain level, making the most reasonable assumption for the edge/screw ratio, i.e. $\psi=\pi / 4$, which amounts to consider equal total lengths of mobile edge and screw dislocation lines in each slip system. Note that this assumption is compatible with the similar mobility that both types of dislocations have in fcc crystals (e.g. [53]). In particular, the simulated texture shown in Figure $4 d$ is the one that most resembles the experimental texture reported at $400^{\circ} \mathrm{C}$ and $10^{-3} \mathrm{~s}^{-1}$. In this case, both threshold stresses for climb and glide were assumed to be equal $\left(\sigma_{o}^{s}=\tau_{o}^{s}\right)$, which resulted in similar contributions from them both. Evidently, the significant fraction of plastic strain accommodated by climb of edge dislocations (which, as discussed above, does not involve lattice rotations) was responsible for a significant retention of the initial (001) cube component. Otherwise, favoring glide over climb by assuming the threshold stress for climb to be twice the glide stress $\left(\sigma_{o}^{s}=2 \times \tau_{o}^{s}\right)$ determines that around $90 \%$ of the strain is accommodated by glide, which in turn predicts a texture (Figure 4e) not far from the glide-only case (Figure 4c). Finally, the fourth row illustrates the importance of using a sound value for the average character of the dislocation density. If a predominant screw dislocation character is assumed (e.g. $\psi=0.01$ ), which amounts to consider a ratio 9:1 between the screw and the edge dislocation densities (a value more compatible with a bcc, rather than a fcc material, due to the much lower mobility of screw dislocation segments in bcc structures [53]), the resulting texture (Figure 4f) is clearly a standard compression texture, with the (110) fiber intensity even higher than the glide-only case. The depletion of the cube component is due to a significant reduction in the edge dislocation climb that tends to stabilize the (001) component. On the other hand, the intensification of the (110) component can be understood regarding the additional simulation shown in Figure $4 \mathrm{~g}$, in which glide-only was assumed, but on slip mode $(112)\langle 110\rangle$, instead of the standard (111) $\langle 110\rangle$ slip. Note that this non-octahedral glide corresponds precisely to glide on planes that are perpendicular to the original (111) slip planes. This simulation corresponds to the slip of screw dislocations onto a slip plane orthogonal to the original slip plane, as described in Section 3.

\section{Concluding remarks}

We propose here a crystallographically based continuum constitutive description of the viscoplastic deformation of single crystals by climb and glide, extending the well known rate-sensitivity approach for crystal plasticity by glide. The model introduces three important constitutive parameters, i.e. the average edge/screw ratio $(\operatorname{tg} \psi)$, the creep exponent associated with climb $\left(n_{c}\right)$ and a threshold stress for climb activation $\left(\sigma_{o}^{s}\right)$. All of them are likely to be temperature-, strain-rate- and microstructure-dependent. Concerning the average character of the dislocations, this quantity is difficult to determine experimentally, since it involves measurements on dislocations moving during the deformation process. However, 3-D dislocation dynamics (DD) models [53-56] may provide useful estimates of the magnitude of this parameter and how it evolves with deformation. Concerning the creep exponent, a value of 3 is widely accepted for thermal creep [5], when this process takes place 
exclusively by climb. However, when creep is controlled by climb but most of the dislocation motion is by glide, the macroscopic creep exponent tends to be higher [1]. The reason for this could be related to the complicated coupling between climb and glide (e.g. when a gliding dislocation stops at an obstacle, a small amount of climb may allow it to overcome the obstacle and continue gliding, providing a significant amount of strain until it reaches the next obstacle, and so on). The proposed model may need the addition of elaborated hardening laws, involving the mutual influence of the glide and climb strains on the evolution of the glide and climb threshold stresses, to capture the above complexity. Finally, the determination of the threshold stress for climb can be either done by inverse analysis, i.e. using available experimental results to adjust the value (or values) of $\sigma_{o}^{s}$ that best fits the data, or using lower length-scale models to obtain the stress needed to move dislocations by climb in the presence of certain local concentration of point defects. This can be done semi-analytically by means of rate theory (e.g. [57]) or, as recently reported by Mordehai et al. [58] (and originally proposed by Raabe [59]), introducing dislocation motion assisted by bulk diffusion in DD models solving the diffusion equation locally for each dislocation segment, or introducing the point defects explicitly in DD codes and solving the elastic interactions between each point defect and the dislocation segments [60].

The two examples provided in the paper show improved predictions of effective behavior and texture development in polycrystalline aggregates deforming in a climb-controlled thermal creep regime. On the other hand, irradiation creep can be treated similarly, although a proper consideration of the chemical component of the climb force is needed to account for the continuous addition of point defects to the material. This determines that the instantaneous point defect concentration will not be in equilibrium during the deformation process, thus affecting the kinetics of dislocation climb and making it necessary to consider the effect of the hydrostatic pressure on the single crystal constitutive behavior. Obviously, such description of climb under irradiation will depend on all the same microstructural variables and applied deformation conditions as in the case of thermal creep, and, additionally, on the type and dose of irradiation, as well as on the particular damaged microstructure developed in the studied material during irradiation [61].

\section{Acknowledgements}

This work was supported by Nuclear Energy Advanced Modeling and Simulation (NEAMS) under the Advance Fuel Cycle Initiative (AFCI) Program, Project \#LA0915010909 (USA). OC acknowledges partial funding from ANR-08-BLAN-00238-02 (France). The authors wish to thank John Hirth and Tom Arsenlis for fruitful discussions.

\section{References}

[1] H.J. Frost and M.F. Ashby, Deformation-Mechanism Maps, Pergamon Press, Oxford, 1982.

[2] J. Weertman, Trans. AIME 218 (1960) p.207. 
[3] J. Weertman, in Rate Processes in Plastic Deformation of Materials, J.C.M. Li and A.K. Mukherjee, eds., American Society for Metals, Metals Park, OH, 1975, p.315.

[4] J. Weertman, J. Appl. Phys. 26 (1955) p.1213.

[5] F.R.N. Nabarro, Phil. Mag. 16 (1967) p.231.

[6] P.T. Heald and M.V. Speight, Phil. Mag. 29 (1974) p.1075.

[7] R. Bullough and J.R. Willis, Phil. Mag. 31 (1975) p.855.

[8] F.R.N. Nabarro, R. Bullough and J.R. Matthews, Acta Metall. 30 (1982) p.1768.

[9] P.A. Turner, C.N. Tomé, N. Christodoulou and C.H. Woo, Phil. Mag. 79 (1999) p.2505.

[10] Y. Rougier, T. Bretheau and A. Zaoui, in Proceedings of SMiRT-12, Stuttgart, K.F. Kussmaul, ed., 1993, p.99.

[11] Y. Rougier, Etude du Comportement sous Irradiation: Modélisation Micromécanique de l'Elastoviscoplasticite, PhD Thesis, Ecole Polytechnique, Palaiseau, France, 1994.

[12] C.N. Tomé and N. Christodoulou, Phil. Mag. 80 (2000) p.1407.

[13] C.S. Hartley, Phil. Mag. 83 (2003) p.3783.

[14] M. Peach and J.S. Koehler, Phys. Rev. 80 (1950) p.436.

[15] J.P. Hirth and J. Lothe, Theory of Dislocations, McGraw-Hill, New York, 1968, p.86.

[16] J. Weertman, Phil. Mag. 11 (1965) p.1217.

[17] J.P. Hirth and J. Lothe, Theory of Dislocations, McGraw-Hill, New York, 1968, p.506.

[18] J. Bardeen and C. Herring, in Imperfections in Nearly Perfect Crystals, W. Shockley, J.H. Hollomon, R. Maurer and F. Seitz, eds., Wiley, New York, 1952, p.261.

[19] R.J. Asaro and A. Neddleman, Acta Metall. 33 (1985) p.923.

[20] U.F. Kocks, A.S. Argon and M.F. Ashby, Prog. Mater. Sci. 18 (1975) p.1.

[21] F.R.N. Nabarro, Acta Mater. 54 (2006) p.263.

[22] R. Becker and S. Panchanadeeswaran, Acta Metall. Mater. 43 (1995) p.2701.

[23] D.P. Mika and P.R. Dawson, Mater. Sci. Eng. A 62 (1998) p.257.

[24] F. Delaire, J.L. Raphanel and C. Rey, Acta Mater. 48 (2001) p.1075.

[25] F. Barbe, L. Decker, D. Jeulin and G. Cailletaud, Int. J. Plasticity 17 (2001) p.513.

[26] D. Raabe, M. Sachtleber, Z. Zhao, F. Roters and S. Zaefferer, Acta Mater. 49 (2001) p.3433.

[27] O. Diard, S. Leclercq, G. Rousselier and G. Cailletaud, Int. J. Plasticity 21 (2005) p.691.

[28] L. Delannay, P.J. Jacques and S.R. Kalidindi, Int. J. Plasticity 22 (2006) p.1879.

[29] A. Musienko, A. Tatschl, K. Schmidegg, O. Kolednik, R. Pippan and G. Cailletaud, Acta Mater. 55 (2007) p.4121.

[30] R.A. Lebensohn, Acta Mater. 49 (2001) p.2723.

[31] R.A. Lebensohn, R. Brenner, O. Castelnau and A.D. Rollett, Acta Mater. 56 (2008) p.3914.

[32] R.A. Lebensohn, M. Montagnat, P. Mansuy, P. Duval, J. Meysonnier and A. Philip, Acta Mater. 57 (2009) p.1405.

[33] P. Van Houtte, S. Li and O. Engler, in Continuum Scale Simulation of Engineering Materials, D. Raabe, F. Roters, F. Barlat and L.Q. Chen, eds., Wiley, Weinheim, 2004, p.459.

[34] A. Molinari, G.R. Canova and S. Ahzi, Acta Metall. 35 (1987) p.2983.

[35] R.A. Lebensohn and C.N. Tomé, Acta Metall. Mater. 41 (1993) p.2611.

[36] C.N. Tomé and R.A. Lebensohn, Viscoplastic Self-Consisitent Source Code (version 7).

[37] R.A. Lebensohn, C.N. Tomé and P. Ponte Castañeda, Phil. Mag. 87 (2007) p.4287.

[38] A.V. Hershey, J. Appl. Mech. 21 (1954) p.236.

[39] R. Masson, M. Bornert, P. Suquet and A. Zaoui, J. Mech. Phys. Solid. 48 (2000) p.1203.

[40] Y.B. Chastel, P.R. Dawson, H.R. Wenk and K. Bennett, J. Geophys. Res. B 98 (1993) p.17757.

[41] P.R. Dawson and H.R. Wenk, Phil. Mag. A. 80 (2000) p.573.

[42] H.R. Wenk and C.N. Tomé, J. Geophys. Res. B 104 (1999) p.25513. 
[43] A. Tommasi, D. Mainprice, G. Canova and Y. Chastel, J. Geophys. Res. B 105 (2000) p.7893.

[44] O. Castelnau, D.K. Blackman, R.A. Lebensohn and P. Ponte Castañeda, J. Geophys. Res. B 113 (2008) p.B09202.

[45] O. Castelnau, D.K. Blackman, R.A. Lebensohn and P. Ponte Castañeda, in Bridging Computational Scales: From Microstructure to Macroscale Properties, O. Cazacu and P. Franciosi, eds., Hermes/Wiley, New York, 2008, p.55.

[46] G. Hirth and D. Kohlstedt, Geophys. Monogr. 138 (2003) p.83.

[47] W.B. Durham and C. Goetze, J. Geophys. Res. 82 (1977) p.5737.

[48] Q. Bai, S.J. Mackwell and D.L. Kohlstedt, J. Geophys. Res. 96 (1991) p.2441.

[49] Q. Bai and D.L. Kohlstedt, Tectonophysics 206 (1992) p.1.

[50] M.G. Stout, S.R. Chen, U.F. Kocks, A.J. Schwartz, S.R. MacEwen and A.J. Beaudoin, in Hot Deformation of Aluminum Alloys II, T.R. Bieler, L.A. Lalli and S.R. MacEwen, eds., TMS, Warrendale, PA, 1998, p.243.

[51] C. Maurice and J.H. Driver, Acta Mater. 45 (1997) p.4627.

[52] D. Raabe, Acta Metall. Mater. 43 (1995) p.1023.

[53] V.V. Bulatov and W. Cai, Computer Simulations of Dislocations, Oxford University Press, Oxford, 2006.

[54] B. Devincre, V. Pontikis, Y. Brechet, G. Canova, M. Condat and L. Kubin, in Microscopic Simulations of Complex Hydrodynamic Phenomena, M. Marechal and B.L. Holian, eds., Plenum Press, New York, 1992, p.413.

[55] H.M. Zbib, M. Rhee and J.H. Hirth, Int. J. Mech. Sci. 40 (1999) p.113.

[56] D. Weygand, L.H. Friedman and E. Van Der Giessen, A. Neddleman, Model. Simul. Mater. Sci. Eng. 10 (2002) p.437.

[57] R. Bullough, M.W. Finnis and M.H. Wood, J. Nucl. Mater. 103/104 (1981) p.1263.

[58] D. Mordehai, E. Clouet, M. Fivel and M. Verdier, Phil. Mag. 88 (2008) p.899.

[59] D. Raabe, Phil. Mag. 77 (1998) p.751.

[60] A. Arsenlis, private communication, 2008.

[61] R.A. Lebensohn, C.N. Tomé, R.A. Hoit and C.S. Hartley, 2010, to be published. 